\title{
Segunda Temporada de Pesca 2016 Los retos de la pesquería industrial de anchoveta
}

\section{Elaborado por la Dirección Científica de Oceana}

1 de diciembre del 2016

\author{
OCEANA $\begin{gathered}\text { Protegeiendolos } \\ \text { oceanos del Mundo }\end{gathered}$
}




\section{Introducción}

Tras 4 días de pesca exploratoria y a 15 días del inicio de la segunda temporada de pesca de anchoveta en la zona norte-centro del litoral, se confirma que la elevada presencia de juveniles representa un importante riesgo y un duro desafío para la pesca industrial del recurso, tal como señaló Oceana en su primera evaluación.

Presentamos un segundo análisis basado en la información proporcionada por el Instituto del Mar del Perú (IMARPE) a través de sus Reportes Diarios de la Actividad Pesquera Industrial. Aunque los datos recogidos por IMARPE no cubren todos los puntos de desembarco, representan una aproximación a los valores oficiales resumidos por el Ministerio de la Producción (PRODUCE), aunque publicados con menor frecuencia.

La cuota de 2 millones de toneladas autorizada para la presente temporada es un valor relativamente alto, considerando que el stock se está recuperando de los impactos oceanográficos del reciente evento El Niño y que su comportamiento reproductivo aún está alterado respecto al patrón histórico. La elevada tasa de juveniles reportados por el crucero 1609-10 de evaluación es la mayor promesa de recuperación del stock: 86\% en número (aprox. 9 de cada 10 individuos) y $49 \%$ en biomasa (aprox. 3.3 millones de toneladas del total estimado en 6.86 para el stock norte centro de anchoveta). Sin embargo, para que esta promesa se concrete es necesario que un alto porcentaje de esta fracción juvenil sobreviva a la temporada de pesca, crezca y se reproduzca. Finalmente, de acuerdo a los indicadores de IMARPE, la etapa reproductiva estaría entrando en su fase de menor actividad. 


\section{Avance de la Temporada de Pesca}

Según los datos de IMARPE, al 28 de noviembre se habrían descargado 380,591 toneladas de anchoveta, lo que representa alrededor del 19\% de la cuota designada para este año. (Figura 1)

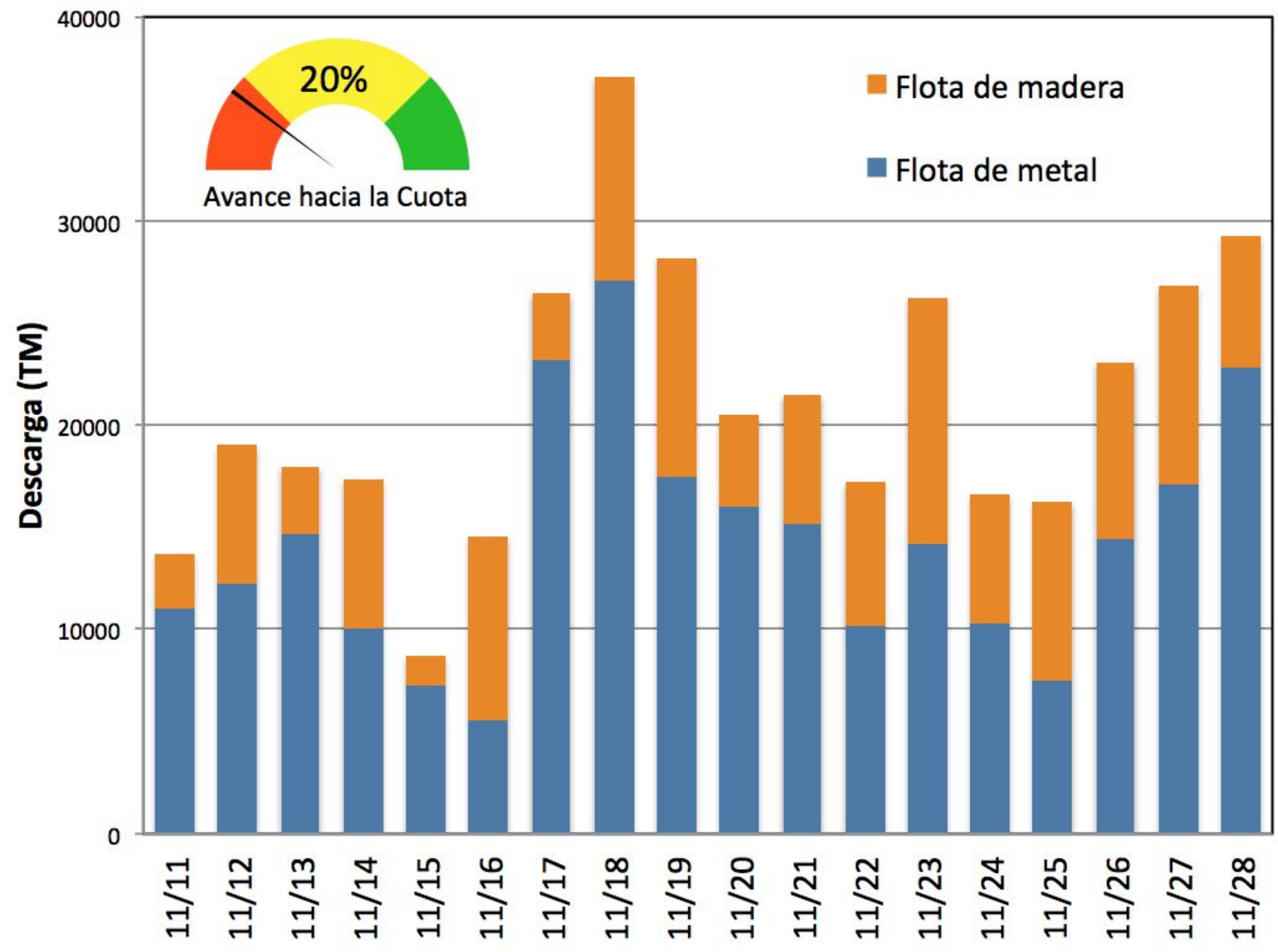

Fecha

Figura 1. Descargas diarias de anchoveta por tipo de flota. Segunda Temporada de Pesca de Anchoveta Stock Norte-Centro 2016. Fuente: IMARPE. Elaboración propia. 
Chimbote encabeza la lista de los puntos de desembarque monitoreados por IMARPE, con una descarga total de 164,792 TM y una descarga diaria promedio de 9,266 TM. EI mayor desembarco registrado hasta el momento corresponde también a este puerto con 19,087 TM el día 18 de noviembre (Figura 2).

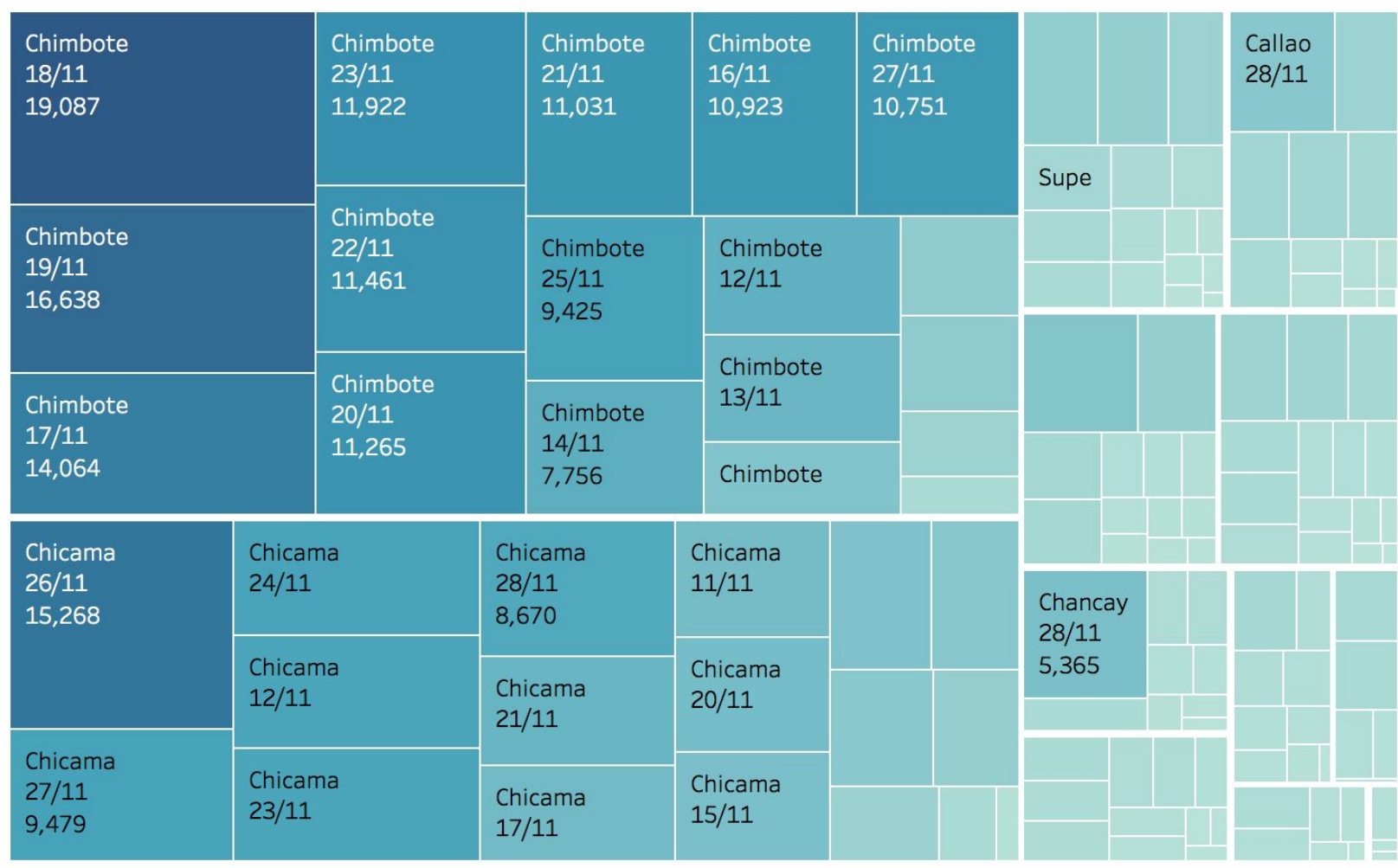

Figura 2. Descargas diarias de anchoveta por puerto ordenadas por volumen de descarga (TM).

Segunda Temporada de Pesca de Anchoveta Stock Norte-Centro 2016. Fuente: IMARPE. Elaboración propia.

Las descargas por embarcación y tipo de flota (metal y madera también conocida como "vikinga") generalmente han sido menores a otras temporadas similares. Los valores promedios diarios para embarcaciones de metal son 96.16 TM y para embarcaciones de madera 38.36 TM. El total de descargas para embarcaciones de metal ha sido de 256,363 TM y para las de madera 124,229 TM (Figura 3). 


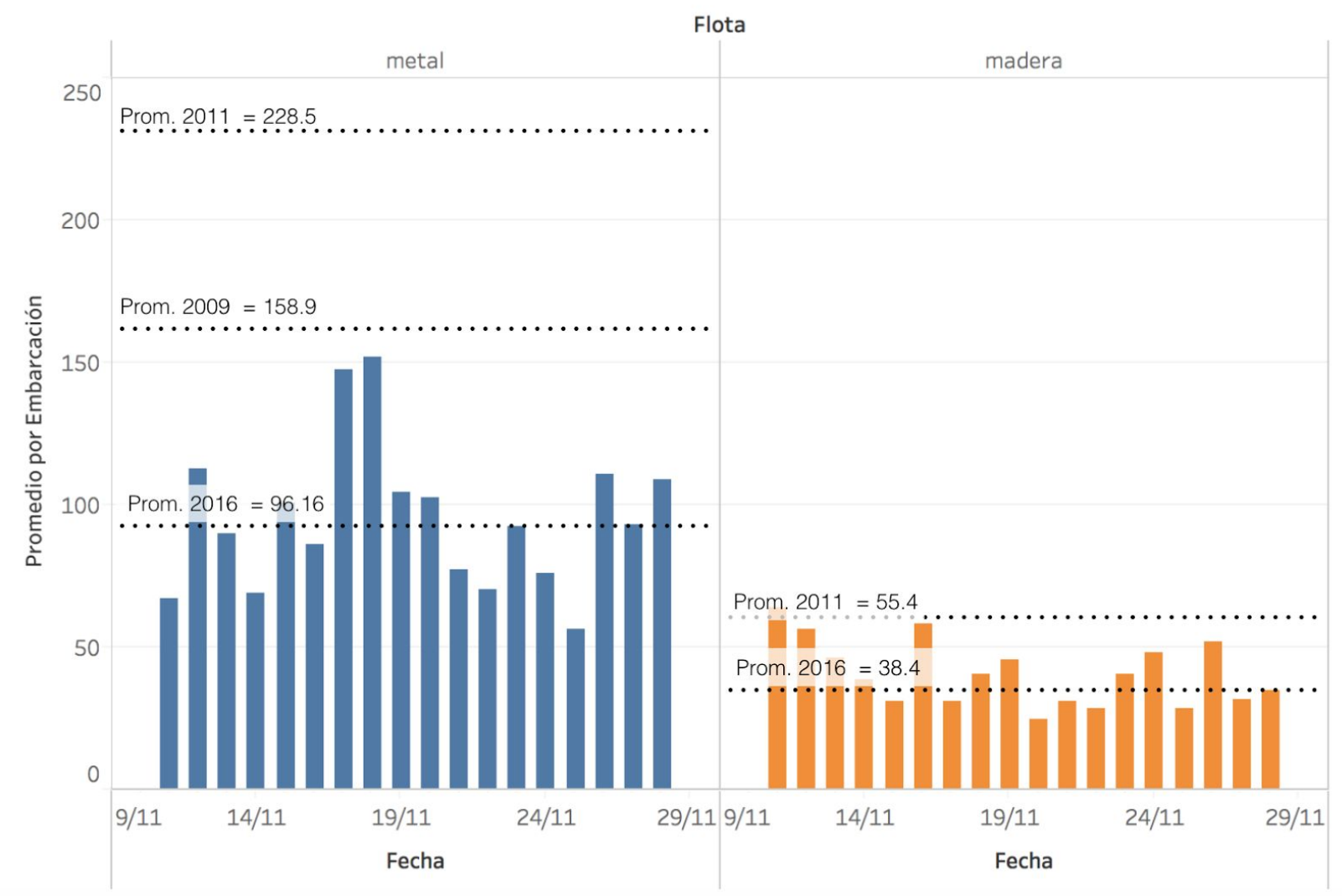

Figura 3. Descargas diarias (TM) de anchoveta por tipo de embarcación de la Segunda Temporada de Pesca de Anchoveta Stock Norte-Centro 2016. Se incluyen como referencia promedios de temporadas anteriores para los primeros 20 días de pesca. Fuente: IMARPE. Elaboración propia.

En términos generales, el avance de la actual temporada en estos primeros días está muy por debajo del promedio de las últimas temporadas. La escasez de cardúmenes grandes y la elevada presencia de juveniles obligó a múltiples cierres preventivos que están afectando el rendimiento de la pesca (Figura 4). 


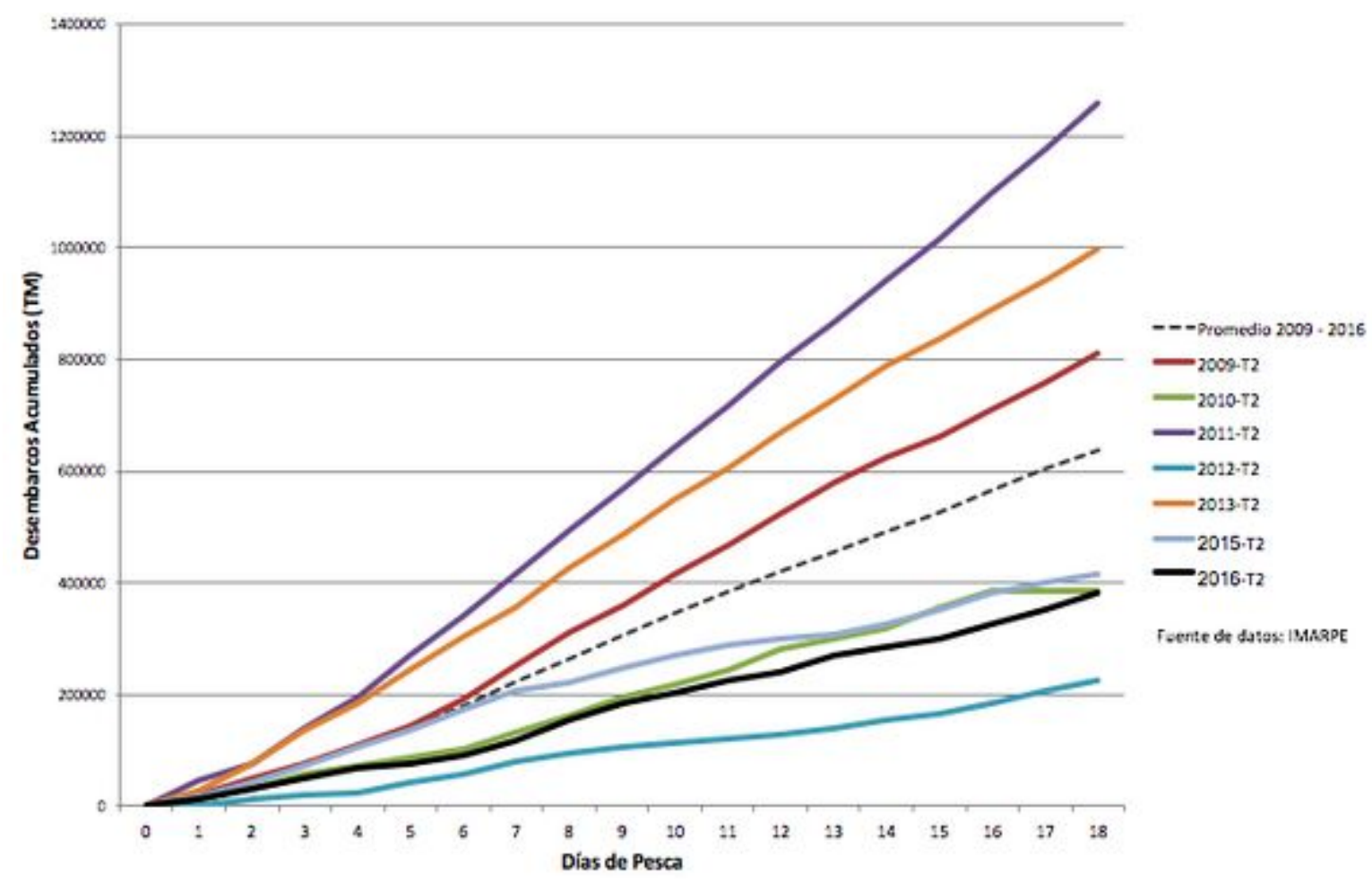

Figura 4. Comparación de las descargas diarias acumuladas de anchoveta para la Segunda Temporada de Pesca de Anchoveta Stock Norte-Centro del 2009 al 2016. Fuente: IMARPE. Elaboración propia.

\section{Pesca de juveniles}

La elevada captura de juveniles ha sido una constante desde inicios de la actual temporada, lo que ha sido evidenciado en los reportes científicos de IMARPE y la advertencia de los pescadores en medios de comunicación. Un panorama previsible, tal como se señaló al inicio de la temporada, que persiste pese a los múltiples esfuerzos del gobierno que a la fecha ha establecido 24 cierres preventivos mediante Resolución Ministerial o comunicados. Esta situación genera dudas sobre la posibilidad de tener una temporada de pesca exitosa, tanto desde el punto de vista económico como biológico, es decir, sin comprometer la recuperación del stock.

La figura 5 presenta los reportes de juveniles de IMARPE, indicando la presencia de juveniles en las descargas de acuerdo a un código de colores para todos los puertos monitoreados con actividad pesquera. Algunos puertos como Chimbote y Huacho han presentado consistentemente valores elevados de juveniles de hasta $97 \%$ el día 24 de noviembre para este último. 


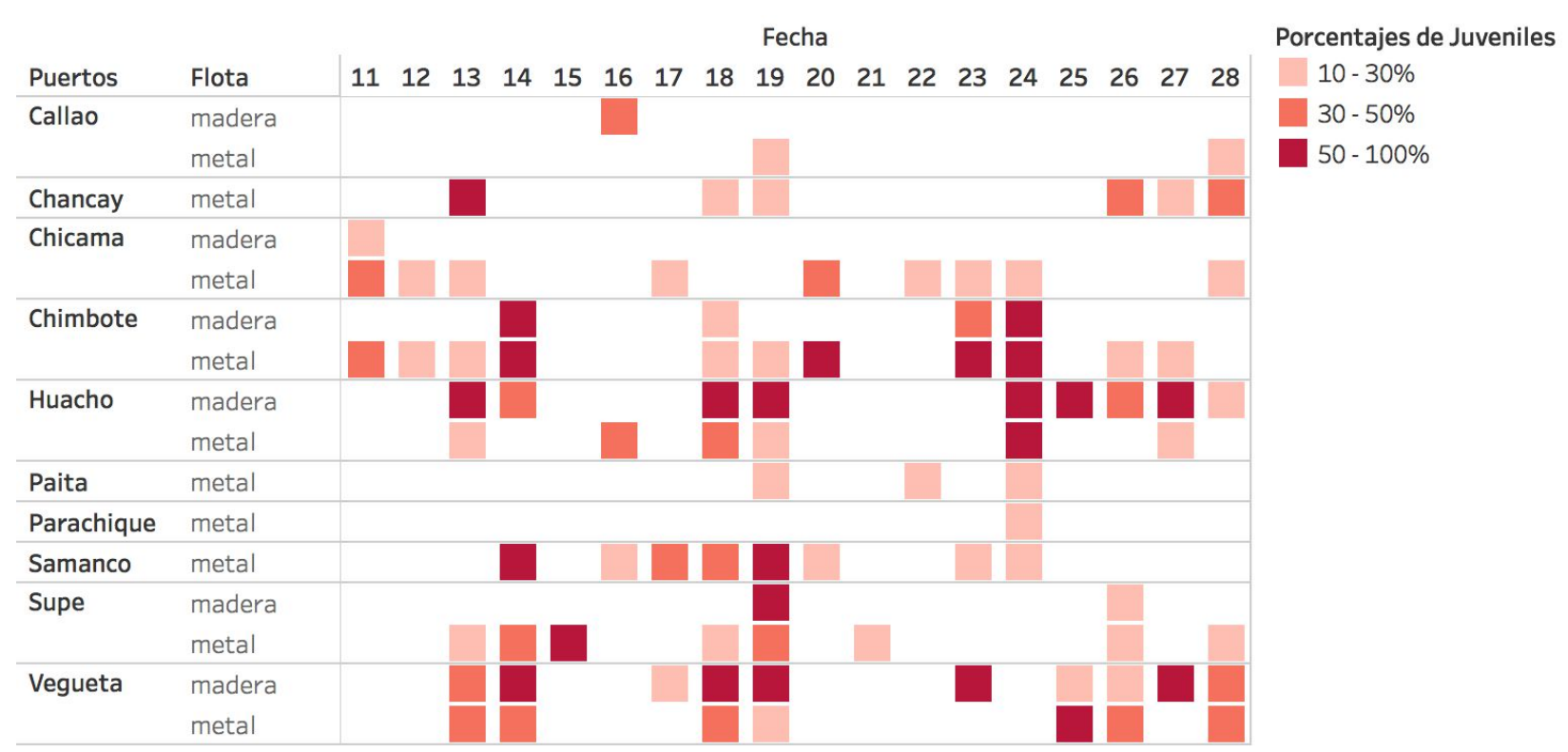

Figura 5. Porcentajes de juveniles de anchoveta por encima del $10 \%$ en desembarcos por puerto y tipo de flota. Segunda Temporada de Pesca de Anchoveta Stock Norte-Centro 2016. Fuente: IMARPE. Elaboración propia.

Cuando se calcula el promedio diario por tipo de flota incluyendo solo los puertos en los cuales se realizó el muestreo de juveniles, se puede observar que los valores son relativamente altos y coinciden con la mayor cantidad de descargas. Es decir, a mayor descarga, mayor frecuencia de juveniles. Esto también concuerda con la gran cantidad de juveniles identificada por IMARPE antes del inicio de la temporada. (Figura 6). 


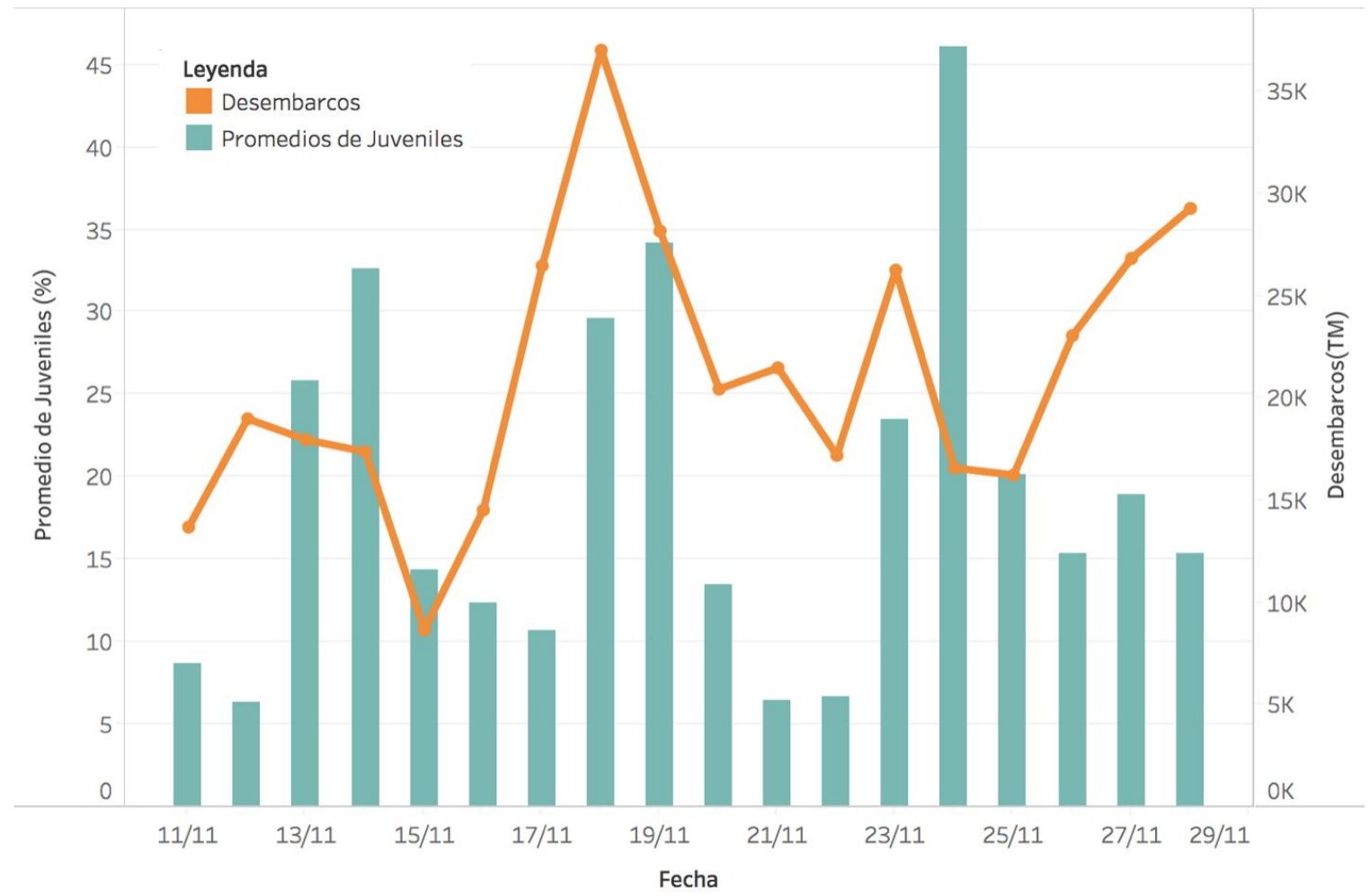

Figura 6. Desembarcos diarios de anchoveta y porcentajes de juveniles mostrando una alta correlación ( $R$ Spearman: -0.628, p:0.00521 ${ }^{* * *}$ ). Segunda Temporada de Pesca de Anchoveta Stock Norte-Centro 2016.

Fuente: IMARPE. Elaboración propia.

\section{Actividad reproductiva}

De acuerdo al último Reporte de Indicadores Reproductivos preparado por IMARPE (No 22 - 30 de noviembre) la anchoveta ya había pasado su pico de desove. Pero como muestra la figura $7 a$, el valor de la fracción desovante es todavía alto aunque por debajo del valor de referencia establecido por IMARPE $(27.7 \%$ para invierno-primavera). En términos prácticos, significa que alrededor de 1 de cada 4 anchovetas todavía se encontraba desovando hacia el inicio de la temporada de pesca.

Adicionalmente, el índice de atresia (un indicador del final del periodo de desove) muestra un valor alto en referencia al valor estandarizado para el periodo 2006-2012, reforzando la idea del término de la temporada reproductiva (Figura 7b). 
Sin embargo, cabe recordar que la especie actualmente se reproduce durante todo el año con un pico reproductivo alto en invierno y otro menor en verano. En estos momentos es posible encontrar hembras con hueveras en regresión (no producirán huevos) que pueden ser identificadas como reproductoras activas y causar alarma entre los pescadores durante las faenas. Lamentablemente, no se han realizado esfuerzos significativos para mejorar la información hacia los gremios pesqueros y su malestar en medios y espacios públicos es evidente.
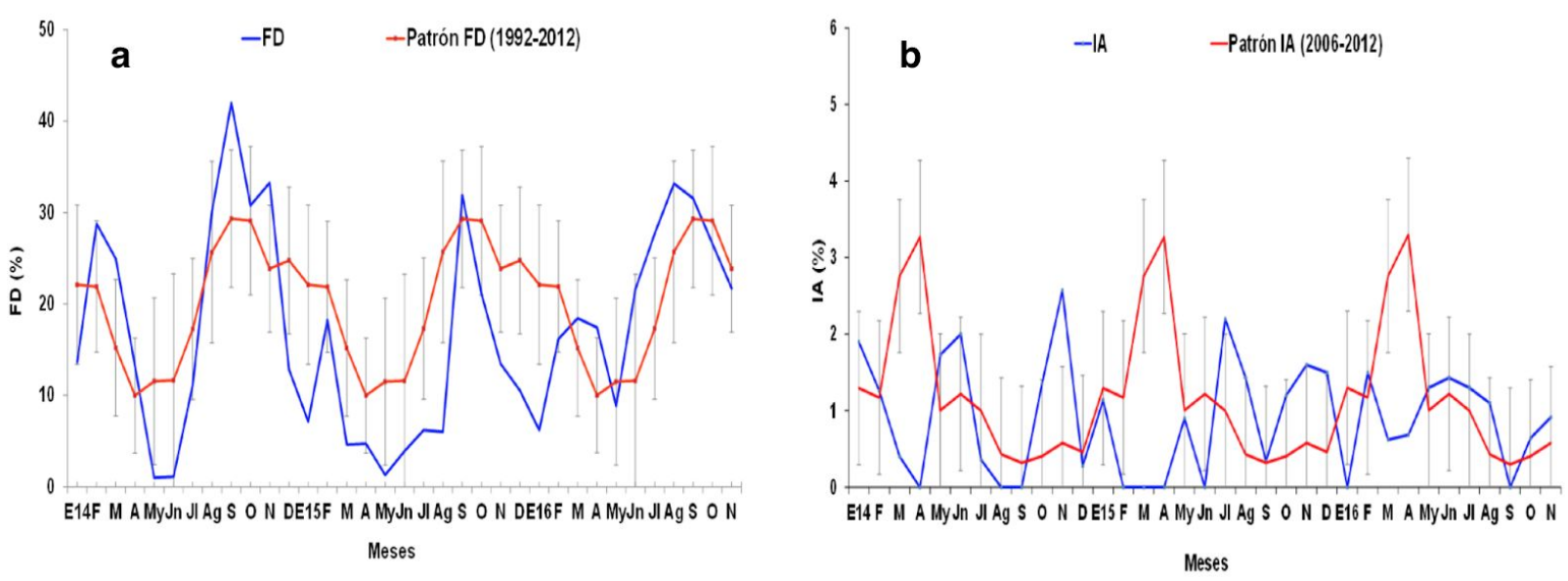

Figura 7. a. Fracción Desovante y b. Índice de Atresia del stock norte-centro de anchoveta indicando el final del periodo principal de desove. Fuente: Reporte de Indicadores Reproductivos de Anchoveta No 22/2016 (Noviembre 30) IMARPE.

\section{Desafíos para el sector pesquero}

Al inicio de la temporada de pesca se dictaron algunas medidas legales dirigidas a reducir los descartes en alta mar de la pesca con juveniles. Con dichas regulaciones se esperaba contar con información oportuna de la presencia de juveniles en el mar y de este modo declarar el cierre de zonas de pesca para prevenir su captura. Hasta la fecha se ha dispuesto el cierre de un total de 24 áreas, algunas de ellas por un periodo de hasta cinco días y abarcando grandes superficies del mar territorial.

De acuerdo a los boletines de PRODUCE, los porcentajes de incidencia de juveniles durante la pesca exploratoria fueron altos (hasta $21.6 \%$ el 14 de noviembre), los que se habrían reducido a alrededor del $12 \%$ conforme se inició la temporada oficial. Esto refleja una oportuna reacción para el cierre de zonas de pesca, pero como se puede apreciar en la figura 6, los cierres también reducen las descargas. El gran reto para el 
sector es desacoplar ambas variables: descarga y presencia de juveniles, para mejorar los rendimientos económicos (los juveniles dificultan las operaciones de pesca, tienen menor contenido graso, etc.) y asegurar la base biológica que sostiene el modelo de negocio actual. Además, se debe considerar el riesgo que corre una embarcación cuando la captura masiva de juveniles le impide recoger la red, ante lo cual, por seguridad elemental, se debe descartar la pesca en el mar. Con los elevados niveles de juveniles esto sería frecuente, además de representar un porcentaje de pesca que no es contabilizada en las estadísticas oficiales.

La disposición del gobierno a compartir información es destacable, pero aún se debe trabajar intensamente para mejorar el acceso a la información de base que sustenta las disposiciones legales. Hasta la fecha, informes claves no han sido puestos a disposición del público en los portales de las entidades involucradas, tal es el caso del reporte de la situación del stock norte-centro al 01 de noviembre de 2016 y la evaluación hidroacústica de recursos pelágicos del Crucero 1609-10.

En ese sentido, la administración anterior tuvo bastante éxito compartiendo reportes de la condición del stock y los cruceros de evaluación de manera oportuna, amplia y pública. Aunque por su naturaleza técnica podrían ser difíciles de leer, siempre se cuenta con la capacidad de la academia y los gremios para comunicarlos a los interesados.

Mención aparte merece IMARPE por el detallado trabajo de producción de reportes de desembarcos a lo largo de la costa, así como la información complementaria de reproducción, producción de huevos, etc. Del mismo modo, la Dirección General de Supervisión y Fiscalización de PRODUCE continúa difundiendo sus Boletines de Pesca, lo cual representa una práctica muy importante para mejorar la transparencia y el acceso a la información.

Por otro lado, se debe mejorar la difusión de la información técnica (biológica, social y económica) sobre la cual se basan las decisiones de pesca, así como propiciar el diálogo con los pescadores y sus gremios. Esta labor puede ser liderada por el gobierno y el sector privado, de modo que los gremios de pescadores tengan acceso oportuno a versiones resumidas de los reportes de IMARPE y un mejor entendimiento de las características particulares que tiene cada temporada de pesca. Hacer oídos sordos a sus reclamos, o peor aún, desacreditarlos, no ayuda a construir lazos de confianza en una actividad en la cual todos deben ser socios en base a intereses comunes. 


\section{Perspectivas}

¿Cuántos juveniles estamos dispuestos a pescar durante esta temporada? Es la gran interrogante. Por un lado, se asume que un $10 \%$ de presencia de juveniles podría ser "aceptable", pero si consideramos una cuota de 2 millones de toneladas estaríamos hablando de entre 200,000 y 230,000 toneladas de juveniles (considerando descartes no reportados). Este valor no sólo representa un potencial riesgo para la recuperación del stock sino que además significa la pérdida de entre 700,000 y un millón de toneladas de biomasa adulta al inicio de la siguiente temporada. De ahí que sea necesario considerar el valor futuro del recurso, además de la inconveniencia biológica de su pesca. Además es necesario que IMARPE presente recomendaciones específicas acerca del volumen de juveniles que puede ser extraído sin comprometer la recuperación del recurso.

Es importante señalar que los cierres preventivos dictaminados PRODUCE, han ayudado a reducir el impacto sobre la fracción de juveniles. Sin embargo, la expectativa de una mayor productividad pesquera al flexibilizar la normatividad para no penalizar su captura y reducir los descartes en el mar, es en estos momentos cuestionable.

En ese sentido y de acuerdo al avance de la temporada, luego de cerca de veinte días de pesca se ha alcanzado apenas el $20 \%$ del total de la cuota y con suerte podríamos alcanzar el $50 \%$ antes del final del año. Lamentablemente, los individuos nacidos durante la primera parte del invierno que actualmente son lo suficientemente pequeños para escapar de las redes, para entonces ya estarían alcanzando el tamaño para ser capturados $y$, por lo tanto, es muy probable que conforme transcurra la temporada aumente el porcentaje de juveniles en los desembarcos.

En síntesis, la perspectiva es poco optimista tanto desde el punto de vista social como económico, pues deja abierta la posibilidad de un cierre temprano de la temporada con la finalidad de reducir la captura de juveniles y mejorar las posibilidades de tener una exitosa y abundante primera temporada de pesca el próximo año. Obviamente, el manejo de la pesquería de una sola especie más grande del mundo en un medio físico cambiante no es una tarea fácil, pero es importante resaltar que diversos actores han tomando conciencia de la necesidad de tomar las debidas precauciones para reducir los riesgos al aprovechar los recursos marinos. 